\title{
Serum osteoprotegerin level, carotid-femoral pulse wave velocity and cardiovascular survival in haemodialysis patients
}

\author{
Gábor Speer $^{1, *}$, Bertalan Cs. Fekete ${ }^{1, *}$, Taha El Hadj Othmane ${ }^{1, *}$, Tamás Szabó ${ }^{2}$, József Egresits ${ }^{1,3}$, \\ Erzsébet Fodor ${ }^{2}$, István Kiss ${ }^{2,3}$, Alexander G. Logan ${ }^{4}$, János Nemcsik ${ }^{2}$, András Szabó ${ }^{1}$, \\ Zsófia K. Németh ${ }^{1}$, Miklós Szathmári ${ }^{1}$ and András Tislér ${ }^{1,2}$ \\ ${ }^{1} 1$ st Department of Medicine, Semmelweis University Budapest, ${ }^{2}$ B. Braun Avitum Nephrological Network, ${ }^{3}$ Division of Angiology \\ and Nephrology, Department of Medicine, St Imre Teaching Hospital, Budapest, Hungary and ${ }^{4}$ Samuel Lunenfeld Research Institute, \\ University of Toronto, Toronto, Canada
}

\begin{abstract}
Background. Osteoprotegerin (OPG) is a marker and regulator of arterial calcification, and it is related to cardiovascular survival in haemodialysis patients. The link between OPG and aortic stiffening - a consequence of arterial calcification - has not been previously evaluated in this population, and it is not known whether OPG-related mortality risk is mediated by arterial stiffening.

Methods. At baseline, OPG and aortic pulse wave velocity (PWV) were measured in 98 chronic haemodialysis patients who were followed for a median of 24 months. The relationship between OPG and PWV was assessed by multivariate linear regression. The role of PWV in mediating OPG related cardiovascular mortality was evaluated by including both OPG and PWV in the same survival model.

Results. At baseline mean (standard deviation) PWV was $11.2(3.3) \mathrm{m} / \mathrm{s}$ and median OPG (interquartile range) was 11.1 (7.5-15.9) $\mathrm{pmol} / \mathrm{L}$. There was a strong, positive, linear relationship between PWV and $\operatorname{lnOPG}(P=0.009$, model $R^{2}=0.540$ ) independent of covariates. During follow-up 23 patients died of cardiovascular causes. In separate univariate survival models both PWV and InOPG were related to cardiovascular mortality [hazard ratios $1.31(1.14-1.50)$ and 8.96 (3.07-26.16), respectively]. When both PWV and lnOPG were entered into the same model, only lnOPG remained significantly associated with cardiovascular mortality [hazard ratio $1.11(0.93-1.33)$ and $7.18(1.89-27.25)$, respectively).

Conclusion. In haemodialysis patients OPG is strongly related to PWV and OPG related cardiovascular mortality risk is, in part, mediated by increased PWV.
\end{abstract}

Correspondence and offprint requests to: Gabor Speer, 1st Department of Medicine, Semmelweis University Budapest, 2/a Korányi S. u., Budapest, H-1083, Hungary. Tel: +36-30-9679-144; Fax: +36-1-313-0250; E-mail: speerga@bel1.sote.hu

*These authors contributed equally to this work and are considered first authors.
Keywords: cardiovascular mortality; osteoprotegerin; pulse wave velocity; vascular calcification

\section{Introduction}

A number of cross-sectional studies reported high prevalence of vascular calcification in haemodialysis (HD) patients [1-4]. In addition, prospective studies demonstrated that vascular calcification of the intima and media of the large arteries is associated with increased risk of cardiovascular events and mortality in this population, independent of classical risk factors [5].

Stiffening of the wall of the aorta- as assessed for example by carotid-femoralis pulse wave velocity (PWV) - has emerged as a robust and independent predictor of all cause and cardiovascular mortality in HD patients [6,7]. Aortic stiffening leads to decreased cushioning function, faster pulse wave propagation and earlier return of the reflected wave to the heart resulting in increased central pulse pressure, increased systolic pressure load to the heart and decreased diastolic pressure to boost coronary circulation $[8,9]$. The positive relationship between the extent of aortic calcification and PWV [10] provides a pathway that is - at least in part - plausible to explain for the cardiovascular morbid events seen in HD patients with arterial calcifications.

Recent studies have indicated that osteoprotegerin (OPG), a soluble decoy receptor of the osteoclast activator RANKL, acts as an important regulatory molecule in vascular disease, such as arterial calcification and atherosclerosis $[11,12]$. Moreover, elevated OPG levels have been associated with the progression of vascular calcification in patients receiving long-term haemodialysis [13], and a recent analysis showed that OPG levels can, in part, explain for the association between coronary artery calcification and chronic kidney disease [14]. OPG is also a novel marker of cardiovascular mortality and clinical events in 
patients with acute myocardial infarction complicated with heart failure [15]. The link between serum OPG levels and cardiovascular survival in HD [16] and renal transplant patients [17] further strengthens the role of OPG as a clinically useful prognostic marker among these patients. While the relationship between OPG and arterial calcification may provide the broad framework to explain for its effects on survival, downstream elements of a possible causal relationship are yet to be identified.

We hypothesize that increased serum OPG levels in HD patients indicate arterial calcification that leads to increased PWV with consequent cardiovascular events. To test this we analysed the relationship between OPG levels and PWV in patients on maintenance HD. Furthermore, we investigated the inter-relationship of PWV and serum OPG levels on cardiovascular mortality in the same cohort during followup.

\section{Subjects and methods}

\section{Patients}

This was a prospective cohort study among 98 chronic HD patients with baseline cross-sectional analysis of the relationship between PWV and OPG levels, and survival analysis for cardiovascular mortality using PWV and OPG as predictor variables during follow-up.

All chronic ( $>3$ months on HD) patients of two dialysis units of a dialysis network were invited to participate. No specific exclusion criteria were applied. Patients who gave written informed consent for participation were included and had baseline clinical assessment, laboratory and PWV measurements and then followed for a median (range) of 24 (0-31) months. Baseline demographic and clinical data were gathered by chart review, and laboratory parameters were measured prior to a midweek dialysis at the time of arterial stiffness assessment. Overt cardiovascular disease was considered to be present if the patient had a documented history of myocardial infarction, revascularization procedure, stroke or peripheral artery disease. Heart failure was not included in the definition of cardiovascular disease as this frequently would have been based on physician assessment only, and signs of hypervolaemia in these HD patients could have led to misclassification of heart failure.

The protocol was approved by the Ethics Committee of the dialysis network and all patients gave written informed consent to their participation.

\section{PWV recordings}

Arterial stiffness was measured as carotid-femoral PWV and carotid augmentation index (AI) using the validated PulsePen tonometer (DiaTecne, Milan, Italy) before a midweek HD session with the patient in the supine position [18]. In each subject two sequences of measurements were performed, and their mean was used for statistical analysis.

The PulsePen device measures the time difference between the R wave of the ECG and the foot of the pulse pressure wave - obtained sequentially above the carotid and the femoral arteries using a handheld tonometer- to calcu- late pulse transit time between these two sites. The average signal of at least 10 heart cycles was used in the measurements at both sites for the assessment of PWV. Surface tape measurement of the distance between the carotid and femoral measurement sites was used to calculate PWV by the PulsePen software. To ensure that alterations in blood pressure and heart rate would not bias the results of PWV assessment, the software of PulsePen automatically rejected measurements in which blood pressure or heart rate changed $>5 \%$ during the time between the sequential carotid and femoral pulse wave recordings.

Carotid tonometric measurements were performed on the side contralateral to the fistula or tunnelled jugular line. The same sides were used to obtain femoral pressure waves. All femoral and carotid pulse pressure wave recordings were evaluated for quality by a single observer (TEHO). Previously we evaluated the intra- and interobserver variability of PWV measurements obtained by the PulsePen device in haemodialysis patients and these were 4.8 and $7.3 \%$, respectively.

Blood pressure and heart rate were recorded by the validated BpTru device (VSM Medtech, Vancouver, Canada) with two sequential measurements manually averaged.

\section{Laboratory measurements}

After the arterial stiffness measurements and prior to dialysis, blood samples were collected for laboratory measurements. Serum OPG levels were measured by ELISA using the commercially available kit by Immundiagnostic AG Bensheim, Germany. Intra- and inter-assay coefficients of variation $(\mathrm{CV})$ were below $8 \%$ and $10 \%$ with a lower detection limit of $0.14 \mathrm{pmol} / \mathrm{L}$. Other parameters were measured by standard laboratory procedures.

\section{Statistical analysis}

To assess baseline association between PWV and OPG linear regression analysis was used. First, we performed univariate analysis using PWV as the outcome and OPG as the predictor variable. The variables considered were the ones listed in Table 1, with the exception of the carotid augmentation index. Next, a multivariate model was constructed that included all other variables besides OPG that showed a significant association with PWV in separate univariate models.

To evaluate the inter-relationship of PWV and OPG levels on cardiovascular mortality Cox proportional hazard regression analyses were used. Cardiovascular mortality was defined as sudden cardiac death, death related to myocardial infarction, arrhythmia, heart failure or stroke as assessed by the attending physician. Follow-up was censored at the time of death from other causes, transplantation, transfer to an other unit or at the end of follow-up on 30 September 2007. During the analysis, first univariate Cox proportional hazards models were used with PWV and OPG levels as predictors to evaluate their separate effects on cardiovascular mortality (Model A). Next, the analyses were repeated by including those variables into both models that showed an association with cardiovascular mortality in separate univariate models (Model B). The variables 
Table 1. Baseline demographic, clinical, haemodynamic and laboratory data of the participants $(n=98)$

\begin{tabular}{|c|c|c|}
\hline Male & $n(\%)$ & $60(61)$ \\
\hline Age & Year & $63.4(14.4)$ \\
\hline Dialysis time $^{\mathrm{a}}$ & Months & $29.6(12.4-48.6)$ \\
\hline Residual diuresis $^{\mathrm{a}}$ & $\mathrm{mL} /$ day & $600(100-1300)$ \\
\hline Body mass index & $\mathrm{kg} / \mathrm{m}^{2}$ & $25.3(4.5)$ \\
\hline Current smoking & $n(\%)$ & $18(18.4)$ \\
\hline Diabetes & $n(\%)$ & $40(40.8)$ \\
\hline Overt cardiovascular disease & $n(\%)$ & $59(60.2)$ \\
\hline Systolic blood pressure & $\mathrm{MmHg}$ & $141.8(23.8)$ \\
\hline Diastolic blood pressure & $\mathrm{MmHg}$ & $77.6(12.1)$ \\
\hline Heart rate & $\mathrm{n} / \mathrm{min}$ & $72.8(13.5)$ \\
\hline carotid-femoral PWV & $\mathrm{m} / \mathrm{s}$ & $11.2(3.3)$ \\
\hline carotid AI & $\%$ & $23.2(12.4)$ \\
\hline Haemoglobin & $\mathrm{g} / \mathrm{L}$ & $113.1(15.5)$ \\
\hline Creatinine & $\mu \mathrm{mol} / \mathrm{L}$ & $677(243)$ \\
\hline Urea & $\mathrm{mmol} / \mathrm{L}$ & $20.7(6.0)$ \\
\hline Cholesterol & $\mathrm{mmol} / \mathrm{L}$ & $4.5(1.6)$ \\
\hline Triglycerides & $\mathrm{mmol} / \mathrm{L}$ & $2.1(1.5)$ \\
\hline HDL-cholesterol & $\mathrm{mmol} / \mathrm{L}$ & $1.2(0.4)$ \\
\hline LDL-cholesterol & $\mathrm{mmol} / \mathrm{L}$ & $2.6(0.9)$ \\
\hline Sodium & $\mathrm{mmol} / \mathrm{L}$ & $137(2.9)$ \\
\hline Potassium & $\mathrm{mmol} / \mathrm{L}$ & $5.22(0.86)$ \\
\hline Calcium & $\mathrm{mmol} / \mathrm{L}$ & $2.29(0.21)$ \\
\hline Phosphor & $\mathrm{mmol} / \mathrm{L}$ & $1.6(0.54)$ \\
\hline Albumin & $\mathrm{g} / \mathrm{L}$ & $39.4(3.99)$ \\
\hline Total protein & $\mathrm{g} / \mathrm{L}$ & $66.9(4.71)$ \\
\hline $\mathrm{PTH}^{\mathrm{a}}$ & $\mathrm{pmol} / \mathrm{L}$ & $7.0(4.0-17.5)$ \\
\hline $25 \mathrm{OH}$ vitamin $\mathrm{D}^{\mathrm{a}}$ & $\mu \mathrm{g} / \mathrm{L}$ & $24.8(18.9-36.1)$ \\
\hline $\mathrm{CRP}^{\mathrm{a}}$ & $\mathrm{mg} / \mathrm{L}$ & $6.8(4.2-12.4)$ \\
\hline $\mathrm{OPG}^{\mathrm{a}}$ & $\mathrm{pmol} / \mathrm{L}$ & $11.1(7.5-15.9)$ \\
\hline
\end{tabular}

Data are mean (SD) or in the case of evidence against normal distribution (a) median (interquartile range) for continuous variables and $n(\%)$ for categorical variables.

AI: augmentation index; PWV: pulse wave velocity; PTH: parathyroid hormone; CRP: C-reactive protein; OPG: osteoprotegerin.

considered were the ones listed in Table 1, with the exception of the carotid augmentation index. Finally, both PWV and OPG were included into the same adjusted model (Model C). If OPG and PWV lie along the same pathway of future morbid cardiovascular events, one expects one or both variables to lose statistical significance.

Data are presented as mean (standard deviation) or in the case of evidence against normal distribution as median (interquartile range) and $n(\%)$ for categorical variables. As serum OPG levels were distributed nonnormally, ln-transformed values were used in the analyses. The SAS statistical package version 6.11 was used in the analyses. $P$-values with a two-sided alpha of 0.05 were considered statistically significant. Hazard ratios (HR) are presented with their $95 \%$ confidence intervals in parentheses.

\section{Results}

There were 126 chronic HD patients at the two dialysis units invited to participate. Of these, 28 patients declined participation leaving 98 patients for inclusion into the study. Baseline demographic, medical, laboratory and haemodynamic information of the participants are presented in Table 1. The most frequent causes for renal disease were vascular-tubulointerstitial (including hypertension) (39\%), diabetes mellitus (33\%) and glomerulonephritis (13\%). Antihypertensive treatment at baseline included beta-blockers in $63 \%$, calcium-channel blockers in $62 \%$, angiotensin receptor blockers or angiotensin-converting enzyme inhibitors in $53 \%$ and alpha-blockers in $30 \%$. Of our patients $62 \%$ were on active vitamin-D therapy and $79 \%$ on calcium carbonate.

PWV measurements were successful in all participants, with a mean predialysis value of $11.2 \mathrm{~m} / \mathrm{s}$ (3.3). There were 12 patients with atrial fibrillation. Their PWV $(11.7 \mathrm{~m} / \mathrm{s})$ did not significantly differ from a dose with regular rhythm $(11.1 \mathrm{~m} / \mathrm{s})$ and the two groups were analysed together.

Results of the univariate and multivariate regression analyses for identifying significant baseline predictors of PWV are presented in Figure 1 and Table 2. In univariate analysis we found a strong positive relationship between PWV and $\operatorname{lnOPG}\left(\beta=3.09, P<0.001, R^{2}=0.23\right)$ (Figure 1$)$. In separate univariate models, other significant predictors of baseline PWV were age, diabetes, history of overt cardiovascular disease, predialysis systolic blood pressure, predialysis creatinine, and sodium and CRP levels. The relationship between PWV and lnOPG remained statistically significant after adjustment for these covariates $(\beta=1.48, P=0.009$, model $R^{2}=0.54$ ) (Table 2 ). There was no relationship between the antihypertensives used and PWV. Furthermore, parameters of mineral metabolism were not significantly related to PWV [calcium $(P=0.190)$, phosphor $(P=0.723)$, $\ln \mathrm{PTH}(P=0.453)$ and $\ln 25 \mathrm{OH}$ vitamin-D $(P=0.791)]$ and these were not included in the multivariate linear regression model.

In separate univariate models, using baseline clinical and laboratorical data as predictors, lnOPG was significantly associated with age, overt cardiovascular disease, body mass index, active vitamin-D therapy, and serum triglyceride, HDL cholesterol and albumin levels. Considering these variables in a multivariate linear regression model, only age $(\beta=0.015, P<0.001)$ and BMI $(\beta=-0.026, P=0.01)$ were significantly related to $\operatorname{lnOPG}\left(\operatorname{model} R^{2}=0.432\right)$.

During follow-up 35 patients died (mortality rate 20.3/100 patient years) of which 23 were due to cardiovascular causes (myocardial infarction: 7, sudden death: 5, arrhythmia: 2, heart failure: 4, stroke: 5). From the remaining 65 patients 6 were transplanted, 1 stopped dialysis due to improvement in renal function, 2 were transferred to other units and data of 54 patients were censored at the end of follow-up. Kaplan-Meier survival curves for cardiovascular mortality using tertiles of PWV and OPG are presented in Figures 2 and 3 ( $\log$ rank $P$-values were 0.034 and 0.0006 , respectively). In separate univariate Cox proportional hazard models the following variables were related to cardiovascular mortality: PWV [HR = $1.31(1.14-1.50)]$, lnOPG [8.96 (3.07-26.16)], age [1.07 (1.03-1.12)], overt cardiovascular disease [3.18 (1.08-9.36)], use of angiotensinconverting enzyme inhibitor or angiotensin II receptor blocker [3.60 (1.35-11.65)] and the haemoglobin level [0.97 (0.95-0.99)]. After adjustment for age, cardiovascular disease, use of angiotensin-converting enzyme inhibitor or angiotensin II receptor blocker and the haemoglobin level in separate multivariate models both PWV and lnOPG remained significantly related to cardiovascular mortality (Table 3, Model B). When however both PWV and InOPG 


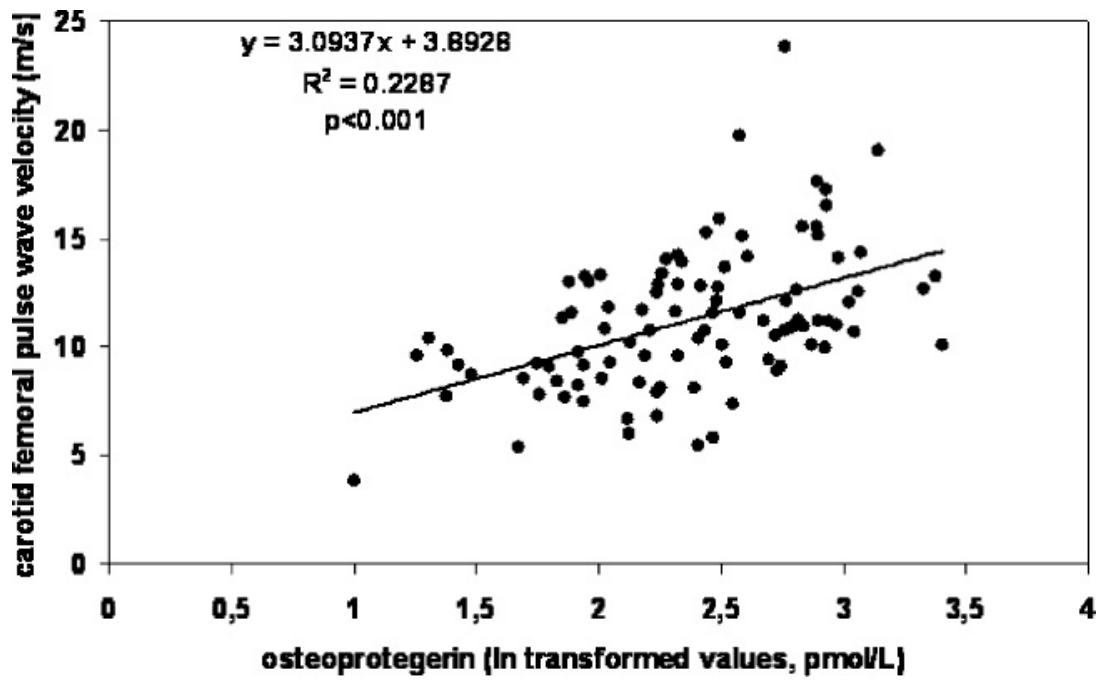

Fig. 1. Linear regression between osteoprotegerin levels and carotid-femoral pulse wave velocity.

Table 2. Significant predictors of carotid-femoral pulse wave velocity in univariate and multivariate linear regression models

\begin{tabular}{|c|c|c|c|c|c|}
\hline \multirow[b]{2}{*}{ Variables } & \multicolumn{2}{|c|}{ Univariate models } & \multirow[b]{2}{*}{$P$-value } & \multicolumn{2}{|c|}{$\begin{array}{l}\text { Multivariate model }{ }^{\mathrm{a}} \\
\left(\text { model } R^{2}=0.54\right)\end{array}$} \\
\hline & Beta & $R^{2}$ & & Beta & $P$-value \\
\hline $\ln$ OPG (1 pmol/L) & 3.094 & 0.23 & $<0.001$ & 1.476 & 0.009 \\
\hline Age $(1$ year $)$ & 0.119 & 0.28 & $<0.001$ & 0.063 & 0.004 \\
\hline Overt cardiovascular disease & 2.738 & 0.16 & $<0.001$ & 1.014 & 0.060 \\
\hline Diabetes mellitus & 2.016 & 0.08 & 0.002 & 0.651 & 0.216 \\
\hline Systolic blood pressure (1 mmHg) & 0.045 & 0.10 & 0.001 & 0.042 & 0.002 \\
\hline $\operatorname{lnCRP}(1 \mathrm{mg} / \mathrm{L})$ & 0.770 & 0.06 & 0.010 & 0.728 & 0.002 \\
\hline Sodium $(1 \mathrm{mmol} / \mathrm{L})$ & -0.267 & 0.05 & 0.017 & -0.134 & 0.113 \\
\hline Creatinine $(100 \mu \mathrm{mol} / \mathrm{L})$ & -0.280 & 0.03 & 0.039 & -0.014 & 0.890 \\
\hline
\end{tabular}

${ }^{a}$ The multivariate linear regression model considers all variables listed in the table.

were included in the same model only lnOPG remained a significant predictor of cardiovascular death (Table 3, Model C).

\section{Discussion}

In this study we demonstrated a strong relationship between PWV and OPG levels and found that the significant predictive value of PWV for cardiovascular mortality is lost when OPG levels are also considered in the same model. These findings may suggest that higher OPG levels and higher PWV are elements of the same pathway that leads from arterial calcification to cardiovascular events and that the mortality risk associated with higher OPG levels may, in part, be explained by increased PWV.

Arterial calcification is a widespread and progressive abnormality in patients on HD that contributes to the high incidence of cardiovascular events [3]. While the exact mechanism linking arterial calcification to cardiovascular events has not been fully evaluated - and it may involve several separate processes - the positive relationship between the degree of aortic calcification and PWV provides one pathway through which arterial calcification can impact on cardiovascular mortality [10]. Indeed, increased carotid-femoral PWV has repeatedly been shown to be an independent predictor of cardiovascular mortality in HD patients [6,7].

Arterial calcification is not a mere consequence of the disturbance of divalent ion metabolism - frequently seen in HD patients - but rather, it is an active process regulated by paracrine and endocrine promoters and inhibitors that are also central to normal bone formation [11]. Among these factors is OPG that recently gained clinical attention. OPG is a glycoprotein that belongs to the tumour necrosis factor (TNF) receptor superfamily, originally discovered as an inhibitor of bone resorption. OPG acts as a decoy receptor of the receptor activator of the nuclear factor $\kappa B$ ligand (RANKL), which is a strong inducer of osteoclast differentiation [12]. OPG knockout mice develop osteoporosis and widespread arterial calcifications implying that OPG is also an inhibitor of vascular calcification [19]. OPG is expressed in vascular cells such as coronary smooth muscle and endothelial cells. Surprisingly, in human crosssectional and follow-up studies, higher rather than lower OPG levels were associated with the degree of coronary and 


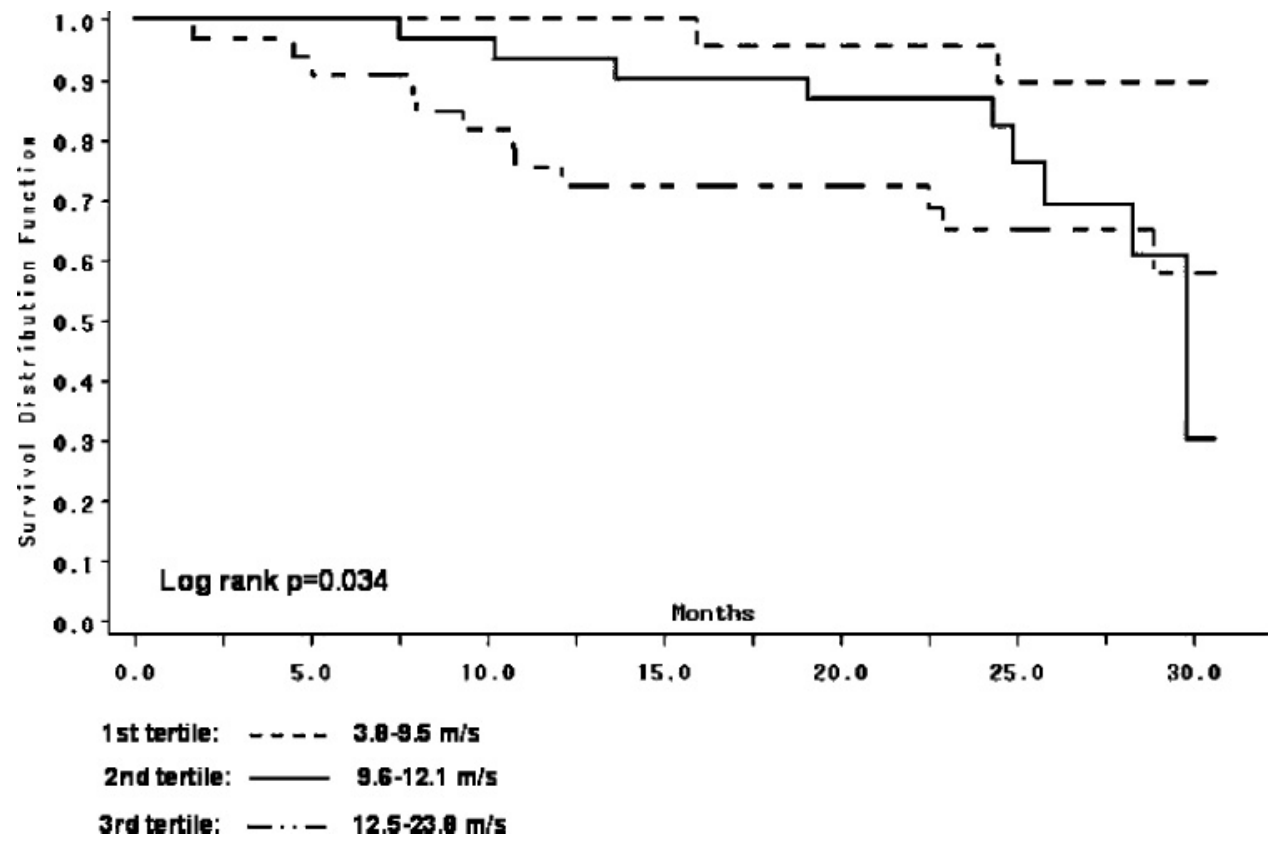

Fig. 2. Kaplan-Meier survival curves for cardiovascular mortality in tertiles of carotid-femoral pulse wave velocity.

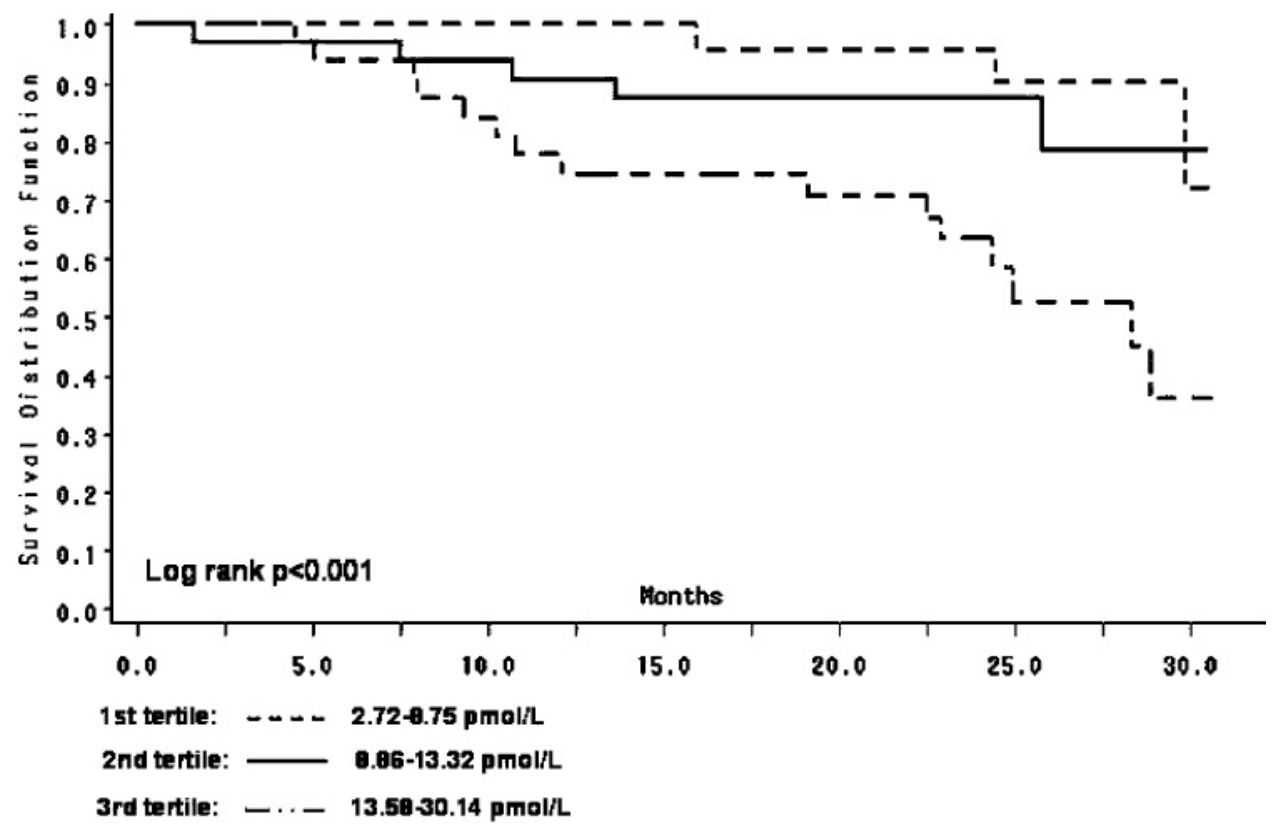

Fig. 3. Kaplan-Meier survival curves for cardiovascular mortality in tertiles of serum osteoprotegerin levels.

aortic calcification [2,20-23], and in HD patients higher rather than lower serum OPG levels predicted progression of aortic calcification [13]. Furthermore, in a recent analysis of non-traditional risk factors, increasing levels of OPG diminished the association between chronic kidney disease and coronary calcification, supporting its direct relationship with calcifications [14]. To explain for the apparent paradox between experimental and human observational data, it has been suggested that increased OPG levels may represent an incomplete defence mechanism against factors that promote atherosclerosis and arterial calcification [22], or alternatively, higher OPG levels may reflect an ongoing attempt of arterial smooth muscle cells to remodel and calcify [24]. The first of these two alternatives was also assumed in postmenopausal osteoporosis, where circulating OPG levels were higher in women with an increased 
Table 3. Association of carotid-femoral pulse wave velocity (PWV) and osteoprotegerin (OPG) levels with cardiovascular mortality

\begin{tabular}{lll}
\hline & \multicolumn{2}{l}{ Hazard ratio (95\% confidence interval) } \\
\cline { 2 - 3 } & PWV & ln OPG \\
\hline Model A $^{\mathrm{a}}$ & $1.31(1.14-1.50)$ & $8.96(3.07-26.16)$ \\
Model B $^{\mathrm{b}}$ & $1.19(1.01-1.41)$ & $8.25(2.22-30.63)$ \\
Model C $^{\mathrm{c}}$ & $1.11(0.93-1.33)$ & $7.17(1.89-27.25)$ \\
\hline
\end{tabular}

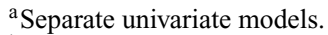

${ }^{\mathrm{b}}$ Separate models adjusted for age, cardiovascular disease, use of angiotensin converting enzyme inhibitor or angiotensin II receptor-blocker and the haemoglobin level.

${ }^{\mathrm{c}}$ Model containing both PWV and lnOPG and adjusted for age, cardiovascular disease, use of angiotensin converting enzyme inhibitor or angiotensin II receptor-blocker and the haemoglobin level.

bone turnover and bone loss. OPG may serve as a protective mechanism to slow down the increased bone resorption and subsequent bone loss [25].

While the exact role of OPG in the development and progression of vascular calcification needs further clarification, the clinical relevance of assessing OPG levels was established by studies demonstrating the predictive value of higher OPG levels for all cause and cardiovascular mortality in HD and renal transplant patients [16,17]. Our finding on the relationship between OPG and PWV provides insight into downstream elements of the association of OPG with cardiovascular events. According to our hypothesis a higher OPG level is a marker of increased arterial calcification and calcification is related to arterial stiffness; therefore OPG is expected to be associated with aortic PWV. While several factors influence arterial stiffness in HD patients such as older age, increased blood pressure, presence of overt cardiovascular disease and inflammation, in our multivariate analysis the relationship between OPG and PWV remained significant after adjustment for these covariates. This finding is compatible with the above hypothesis.

Two previous studies reported on the analysis of the association between OPG and PWV. Kim et al. in their cross-sectional study of newly diagnosed diabetic patients and controls found a significant correlation between serum OPG levels and brachial-ankle PWV [26]. More recently, Stompor et al. reported a significant positive correlation between serum OPG levels and aortic PWV in patients on peritoneal dialysis [27]. Our results add to these findings, as none of the above studies used multivariate analysis to identify an independent association between OPG and PWV, and none of them followed the patients in an attempt to evaluate the inter-relationship of these parameters on clinical outcomes.

In the follow-up phase of our study we found that in separate multivariate survival models both PWV and OPG levels predicted CV mortality, independent of covariates. This confirms previous studies on the prognostic value of these parameters in HD patients and provides external validity to our data. When OPG and PWV were considered in the same survival model, only OPG remained significantly related to future cardiovascular death. This result suggests that the OPG level and PWV provide overlapping prognostic infor- mation in keeping with our hypothesis that they represent a common pathway leading to cardiovascular mortality.

Although antihypertensive drug treatment influences arterial stiffness [28], in our study we found no association between the use of different antihypertensive medications and PWV. Failure to find such a relationship is likely due to the cross-sectional nature of our analysis on the determinants of baseline PWV that does not exclude the possibility of antihypertensives impacting on PWV.

We found no association between parameters of mineral metabolism and PWV. While abnormalities in these variables contribute to arterial calcification, their participation in the development of arterial stiffness in renal patients has not yet been demonstrated clearly and constantly, with some studies showing an association [10,29] while others not [30]. It may be that a snapshot of phosphate or PTH levels is insufficient to describe the abnormality in mineral metabolism, particularly if one considers the time needed to exert an effect on arterial stiffness that may not be apparent in a cross-sectional analysis.

The use of angiotensin-converting enzyme inhibitors or angiotensin receptor blockers was related to cardiovascular mortality in our survival analysis. This surprising finding is likely explained by the strong correlation between the presence of cardiovascular disease at baseline and the use of these drugs (that indicates higher baseline risk in these patients).

Two of three tertile survival curves in each figure superimpose after 30 months. This is likely explained by the widening confidence intervals of the survival curves due to decreasing number of patients at risk by time.

The limitations of our study need to be addressed. First, our data do not provide an explanation for the cross-sectional relationship between OPG levels and PWV. While the known relationship of arterial calcification to both of these factors could explain our finding, it is to be acknowledged that in our study we had no direct assessment of arterial calcification. Alternative explanation for the relationship of OPG with PWV such as the protective effects of OPG on the endothelial cell survival should also be considered. Second, the $95 \%$ confidence interval for the hazard ratio of PWV in the survival analysis when both PWV and OPG were considered (Model C) was wide; therefore we cannot exclude the possibility that PWV is indeed related to cardiovascular mortality independent of OPG.

In summary, in this study we found significant positive relationship between serum OPG levels and aortic PWV in patients on HD and demonstrated an inter-relationship of these parameters on their effect on cardiovascular mortality. These findings are compatible with the hypothesis that the prognostic significance of high OPG levels is, at least in part, mediated by higher PWV in these patients. Whether this relationship is dependent on the degree of aortic calcification and whether the same relationship holds true in other populations as well need further study.

Acknowledgements. The study was supported by research grants from the Hungarian Society of Hypertension and the Hungarian Society of Nephrology.

Conflict of interest statement. None declared. 


\section{References}

1. Ketteler M, Schlieper G, Floege J. Calcification and cardiovascular health: new insights into an old phenomenon. Hypertension 2006; 47 : 1027-1034

2. Barreto DV, Barreto FC, Carvalho $\mathrm{AB}$ et al. Coronary calcification in hemodialysis patients: the contribution of traditional and uremiarelated risk factors. Kidney Int 2005; 67: 1576-1582

3. Caplin B, Wheeler DC. Arterial calcification in dialysis patients and transplant recipients. Semin Dial 2007; 20: 144-149

4. Sigrist M, Bungay P, Taal MW et al. Vascular calcification and cardiovascular function in chronic kidney disease. Nephrol Dial Transplant 2006; 21: 707-714

5. London GM, Guérin AP, Marchais SJ et al. Arterial media calcification in end-stage renal disease: impact on all-cause and cardiovascular mortality. Nephrol Dial Transplant 2003; 18: 1731-1740

6. London GM, Marchais SJ, Guerin AP et al. Arterial stiffness: pathophysiology and clinical impact. Clin Exp Hypertens 2004; 26: 689-699

7. London GM, Cohn JN. Prognostic application of arterial stiffness task forces. Am J Hypertens 2002; 15: 754-758

8. Laurent S, Cockroft J, Van Bortel L et al. Expert consensus document on arterial stiffness: methodological issues and clinical applications. Eur Heart J 2006; 27: 2588-2605

9. Gusbeth-Tatomir P, Covic A. Causes and consequences of increased arterial stiffness in chronic kidney disease patients. Kidney Blood Press Res 2007; 30: 97-107

10. Raggi P, Bellasi A, Ferramosca E et al. Association of pulse wave velocity with vascular and valvular calcification in hemodialysis patients. Kidney Int 2007; 71: 802-807

11. Giachelli CM. Vascular calcification mechanisms. J Am Soc Nephrol 2004; 15: 2959-2964

12. Rogers A, Eastell R. Circulating osteoprotegerin and receptor activator for nuclear factor kappaB ligand: clinical utility in metabolic bone disease assessment. J Clin Endocrinol Metab 2005; 90: 6323-6331

13. Nitta K, Akiba T, Uchida K. The progression of vascular calcification and serum osteoprotegerin levels in patients on long-term hemodialysis. Am J Kidney Dis 2003; 42: 303-309

14. Baber U, de Lemos JA, Khera A et al. Non-traditional risk factors predict coronary calcification in chronic kidney disease in a populationbased cohort. Kidney Int 2008; 73: 615-621

15. Ueland T, Jemtland R, Godang K et al. Prognostic value of osteoprotegerin in heart failure after acute myocardial infarction. $\mathrm{J}$ Am Coll Cardiol 2004; 44: 1970-1976

16. Morena M, Terrier N, Jaussent I et al. Plasma osteoprotegerin is associated with mortality in hemodialysis patients. J Am Soc Nephrol 2006; 17: 262-270
17. Hjelmesaeth J, Ueland T, Flyvbjerg A et al. Early posttransplant serum osteoprotegerin levels predict long-term (8-year) patient survival and cardiovascular death in renal transplant patients. J Am Soc Nephrol 2006; 17: 1746-1754

18. Salvi P, Lio G, Labat C et al. Validation of a new non-invasive portable tonometer for determining arterial pressure wave and pulse wave velocity: the PulsePen device. J Hypertens 2004; 22: 285-293

19. Bucay N, Sarosi I, Dunstan CR et al. Osteoprotegerin-deficient mice develop early onset osteoporosis and arterial calcification. Genes Dev 1998; 12: 1260-1268

20. Jono S, Ikari Y, Shioi A et al. Serum osteoprotegerin levels are associated with the presence and severity of coronary artery disease. Circulation 2002; 106: 1192-1194

21. Abedin M, Omland T, Ueland T et al. Relation of osteoprotegerin to coronary calcium and aortic plaque (from the Dallas Heart Study). Am J Cardiol 2007; 99: 513-518

22. Anand DV, Lahiri A, Lim E et al. The relationship between plasma osteoprotegerin levels and coronary artery calcification in uncomplicated type 2 diabetic subjects. J Am Coll Cardiol 2006; 47: 18501857

23. Mazzaferro S, Pasquali M, Pugliese F et al. Serum levels of calcification inhibition proteins and coronary artery calcium score: comparison between transplantation and dialysis. Am J Nephrol 2007; 27: 7583

24. Moe SM, Reslerova M, Ketteler M et al. Role of calcification inhibitors in the pathogenesis of vascular calcification in chronic kidney disease (CKD). Kidney Int 2005; 67: 2295-2304

25. Mezquita-Raya P, de la Higuera M, García DF et al. The contribution of serum osteoprotegerin to bone mass and vertebral fractures in postmenopausal women. Osteoporosis Int 2005; 16: 1368 1374

26. Kim SM, Lee J, Ryu OH et al. Serum osteoprotegerin levels are associated with inflammation and pulse wave velocity. Clin Endocrinol (Oxf) 2005; 63: 594-598

27. Stompór T, Krzanowski M, Kusnierz-Cabala B et al. Pulse wave velocity and proteins regulating vascular calcification and bone mineralization in patients treated with peritoneal dialysis. Nephrol Dial Transplant 2006; 21: 3605-3606

28. Mahmud A. Reducing arterial stiffness and wave reflection-quest for the holy grail? Artery Research 2007; 1: 13-19

29. London GM, Guérin AP, Verbeke FH et al. Mineral metabolism and arterial functions in end-stage renal disease: potential role of 25 hydrocivitamin D. J Am Soc Nephrol 2007; 18: 613-620

30. Haydar AA, Covic A, Colhoun $\mathrm{H}$ et al. Coronary artery calcification and aortic pulse wave velocity in chronic kidney disease patients. Kidney Int 2004; 65: 1790-1794

Received for publication: 30.1 .08

Accepted in revised form: 8.4 .08 